\title{
The broadband microwave spectra of the monoterpenoids thymol and carvacrol: Conformational landscape and internal dynamics
}

\author{
D. Schmitz, ${ }^{1,2}$ V. A. Shubert, ${ }^{1,2}$ B. M. Giuliano, ${ }^{3, a)}$ and M. Schnell ${ }^{1,2,4, b)}$ \\ ${ }^{1}$ Max Planck Institute for the Structure and Dynamics of Matter, Hamburg, Germany \\ ${ }^{2}$ The Center for Free-Electron Laser Science, Hamburg, Germany \\ ${ }^{3}$ Center for Astrobiology, INTA-CSIC, Torrejón de Ardoz, Madrid, Spain \\ ${ }^{4}$ The Hamburg Centre for Ultrafast Imaging, Universität Hamburg, Hamburg, Germany
}

(Received 16 April 2014; accepted 25 June 2014; published online 17 July 2014)

\begin{abstract}
The rotational spectra of the monoterpenoids thymol and carvacrol are reported in the frequency range $2-8.5 \mathrm{GHz}$, obtained with broadband Fourier-transform microwave spectroscopy. For carvacrol four different conformations were identified in the cold conditions of the molecular jet, whereas only three conformations were observed for thymol. The rotational constants and other molecular parameters are reported and compared with quantum chemical calculations. For both molecules, line splittings due to methyl group internal rotation were observed and the resulting barrier heights could be determined. The experimental barrier heights, $4.0863(25) \mathrm{kJ} / \mathrm{mol}$ for trans-carvacrol-A, $4.4024(16) \mathrm{kJ} / \mathrm{mol}$ for trans-carvacrol-B, and $0.3699(11) \mathrm{kJ} / \mathrm{mol}$ for trans-thymol-A, are compared with similar molecules. @ 2014 AIP Publishing LLC. [http://dx.doi.org/10.1063/1.4887337]
\end{abstract}

\section{INTRODUCTION}

Terpenes represent one of the largest classes of secondary metabolites in nature and are derived from adding substituents to their core building block, isoprene. They exhibit a huge assortment of structures and thus a variety of chemical and biological activities. Furthermore, the natural emission of terpenes by plants contributes significantly to the amount of secondary organic aerosols in the atmosphere. These emissions have implications on atmospheric chemistry and ultimately Earth's climate., ${ }^{1,2}$ Despite their importance, only a few high-resolution spectroscopic studies of terpenes have been reported. Nguyen et al. recently published the first microwave spectroscopic investigation of a linear monoterpene, linalool. ${ }^{3}$ Of the monocyclic monoterpenes, only perillaldehyde, ${ }^{4}$ carvone, and limonene ${ }^{5}$ have been investigated with high-resolution microwave spectroscopy. These studies are in line with previous work on medium-sized biologically relevant molecules (e.g., monosaccharides, ${ }^{6}$ amino acids, ${ }^{7-9}$ nucleobases, ${ }^{10,11}$ neurotransmitters, ${ }^{12}$ and drugs $^{13-15}$ ) that demonstrate the suitability of microwave spectroscopy for obtaining information about the molecular structure, conformational preferences, and internal dynamics of this class of molecules.

In the present investigation, we report the vibrationally and rotationally cold gas-phase microwave spectra of the monocyclic monoterpenes and structural isomers, thymol and carvacrol. For both molecules, cis- and trans-conformational isomers can exist and the corresponding structures are depicted in Figure 1. Thymol and carvacrol differ in the position of the hydroxyl group, which impacts the flexibil-

\footnotetext{
a) Present address: Chemistry Department "G. Ciamician," University of Bologna, Bologna, Italy.

b) Author to whom correspondence should be addressed. Electronic mail: melanie.schnell@mpsd.mpg.de
}

ity of both the hydroxyl and the methyl groups. Due to their anti-bacterial, ${ }^{16-18}$ anti-inflammatory, ${ }^{19}$ and anti-fungal properties ${ }^{20}$ thymol and carvacrol are particularly interesting for the pharmacological and foodstuff industries. They occur naturally as the main constituents in the essential oils of thyme, oregano, and satureja.

Spectroscopically interesting is the influence of the hydroxyl group on the ability of the methyl top to rotate internally. Microwave spectroscopy is well suited to observe the effects of large amplitude motion, e.g. internal rotation, and enables the determination of the corresponding rotational energy barrier. Since the chemical environment of the moving group differs between different molecules, and even between conformers of the same molecule, the derivation of a universal description of internal rotation is challenging. Recent improvements of the theoretical description of different barrier types show that development in this field is still highly active. ${ }^{15,21-23}$ Herein, we discuss the influence of the hydroxyl group on the barrier to methyl group internal rotation, which can be expected to be largely different for thymol and carvacrol, and compare the results to structurally similar molecules, such as $o$ - and $m$-cresol. ${ }^{24,25} \mathrm{We}$ also determine the influence of the hydroxyl group on the configuration of the isopropyl group and hence the conformational preferences, as well as address the hydrogen bonds involved.

The paper is organized as follows: We first briefly describe the experimental setup and the quantum chemical calculations, followed by presentation of the experimental and computational results for each of the molecules under study. We investigate the conformational space of the molecules and the barriers to internal rotation of the methyl tops. Subsequently, we discuss and compare the results of thymol and carvacrol to each other and with literature data. Finally, we summarize the findings and give a short outlook. 


\section{EXPERIMENTAL AND COMPUTATIONAL METHODS}

Thymol (2-isopropyl-5-methylphenol, 99.5\% purity) and carvacrol (5-isopropyl-2-methylphenol, 98\% purity) were purchased from Sigma-Aldrich Chemie $\mathrm{GmbH}$, Steinheim, Germany and were used without further purification. Thymol is a white crystalline powder with a stated melting point of $48-51^{\circ} \mathrm{C}$ and a boiling point of $232{ }^{\circ} \mathrm{C}$. Carvacrol is a yellowish liquid with a stated melting point of $3-4^{\circ} \mathrm{C}$ and a boiling point of $237^{\circ} \mathrm{C}$. The chemical structures of both molecules are depicted in Figure 1. The orientation of the OH-group is defined with respect to the $\mathrm{C} 2$ atom of the benzene ring and its rotation gives rise to trans- and cis-conformers for both molecules.

All rotational spectroscopy measurements were performed with the Hamburg COMPACT spectrometer which has been detailed elsewhere ${ }^{26}$ and thus only a brief description is given here. After supersonic expansion into the vacuum chamber, the ensemble of molecules was polarized with a $1 \mu$ s chirp spanning $2 \rightarrow 8.5 \mathrm{GHz}$. This frequency range perfectly fits the expected high-intensity transitions of internally cold molecules with the size of thymol and carvacrol. The chirp was generated with an arbitrary waveform generator (AWG), amplified to $300 \mathrm{~W}$ with a traveling wave tube amplifier, and finally transmitted into the vacuum chamber with a horn antenna. Following excitation, the free induction decay (FID) of the macroscopic ensemble of polarized molecules was recorded. For each spectrum, $50 \mu \mathrm{s}$ of the FID were recorded at a resolution of $10 \mathrm{ps}$, yielding a frequency resolution of $20 \mathrm{kHz}$ in the Fourier transform (FT) and hence the rotational spectra obtained. Direct triggering of the oscilloscope by the AWG ensures phase stability and permits flexibility for the delay generator specifications. The molecules were seeded into a supersonic expansion using a pulsed nozzle (Parker General Valve, Series 9) operating at $2 \mathrm{~Hz}$ to create a cold molecular jet. The sample holders were placed directly prior to the nozzle and heated to $100^{\circ} \mathrm{C}$ or $140^{\circ} \mathrm{C}$ for thymol or carvacrol, respectively. A constant flow of neon at stagnation pressures of 3.0 bars transported the molecules to the nozzle. Note that the tubing between the reservoir and the valve, as well as the valve itself, was heated to avoid condensation. For carvacrol and thymol, 300000 FIDs and 438000 FIDs, respectively, were co-added for each spectrum. After performing the Fourier transformation, residual background lines were removed by masking, which means that the intensities in the spectrum at the frequencies identified as background lines were set to zero. We used different approaches to identify background lines in our spectrum: (1) by a comparison of the spectra using only the first and only the last $20 \mu \mathrm{s}$ of the FID, where the molecular signal is already significantly reduced; (2) by an individual background measurement with the valve switched off; (3) by a comparison of the two measurements of thymol and carvacrol.

One of the primary advantages of microwave spectroscopy is its ability to obtain information about the gasphase structure of molecules to a high precision. However for a complete structure determination, rotational constants from numerous different isotopologues are required. Such an effort was recently reported for a comparatively large molecule, strawberry aldehyde $\left(\mathrm{C}_{12} \mathrm{H}_{14} \mathrm{O}_{3}\right) \cdot{ }^{27}$ However, this approach remains experimentally demanding and time intensive. Another way to extract structural information from the experimental data is comparison with quantum chemical calculations. The assignment of an experimentally obtained spectrum (e.g., by comparison of rotational constants, barrier to internal rotation, dipole moments) to a quantum chemically optimized structure has given good results, even for larger molecules and complexes. ${ }^{28}$

Herein, the experimental results were compared with electronic structure calculations performed with the Gaussian $03^{29}$ and Gaussian $09^{30}$ program suites. In order to determine the preferred orientations of the isopropyl groups, we carried out relaxed energy scans of the potential energy surface (PES) of the isopropyl group rotation. As starting structures for the energy scans, we used the cis- and trans-conformers of thymol and carvacrol as depicted in Figure 1. We employed the Becke, three parameter, Lee-Yang-Parr (B3LYP) exchangecorrelation functional with the $6-311++G(d, p)$ basis set for these scans. We found two unequal minima in the PES for each starting structure. To further refine the structures and

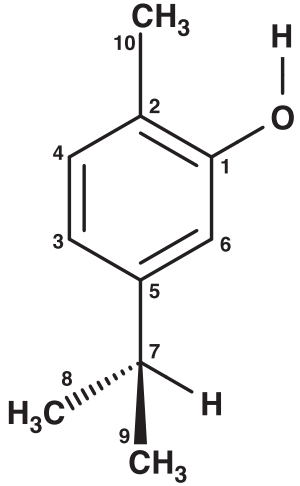

(a) cis-carvacrol

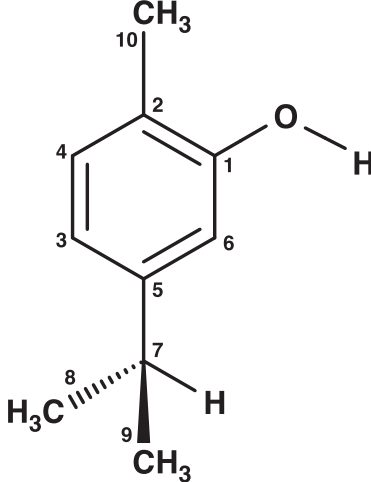

(b) trans-carvacrol

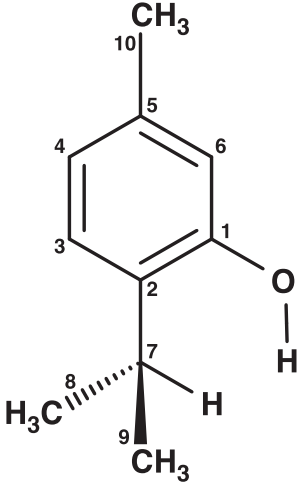

(c) cis-thymol

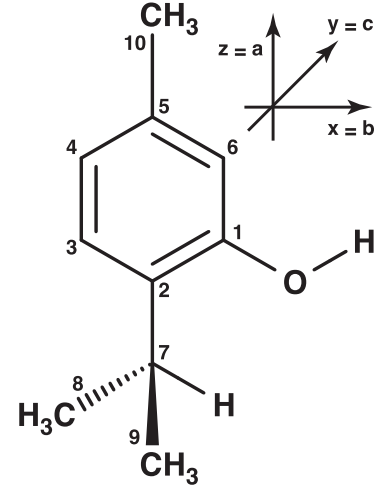

(d) trans-thymol

FIG. 1. Schemes of the structures of the aromatic monoterpenoids thymol and carvacrol. They differ in the position of the hydroxyl substituent with respect to the isopropyl and the methyl groups. The coordinate system next to trans-thymol represents an approximation of the principal axes, which varies slightly between the different molecules and conformations. When discussing the orientation of the methyl rotor, the orientation of the axes with respect to the rest of the molecule becomes important. 
verify the energy ordering of the individual conformations, optimizations and harmonic frequency calculations were carried out on all minima using several different basis sets and methods: B3LYP/6-311++G(d,p), B3LYP/aug-cc-pVTZ, MP2/6-311++G(d,p) (Möller-Plesset second order perturbation theory) and M06-2X/6-311++G(d,p) (Minnesota functional). In order to get an overview of the internal dynamics, the cis-trans isomerization barrier was also computed, as well as the barrier to internal rotation for the different methyl tops. These calculations were performed at the B3LYP/6$311++G(d, p)$ level of theory for selected minima. Here, the barriers to internal rotation for the methyl tops at positions five and two of thymol and carvacrol, respectively, are particularly interesting because they are expected to be similar to that of $o$ - and $m$-cresol. ${ }^{24,25}$ The barrier to internal rotation of those molecules is small enough that the corresponding splitting of the lines into $\mathrm{A}$ and $\mathrm{E}$ species components of the rotational transitions should be observed in our spectra.

\section{RESULTS}

\section{A. Carvacrol}

We first present the computational findings and use them to complement and interpret the experimental results. The relaxed PES of the isopropyl group rotation for the cis- and trans-conformers are depicted in Figure 2. The potential energy relative to the global minimum (denoted as trans-B) is plotted as a function of the dihedral angle, H-C7-C5-C6. The trans-carvacrol PES is lower in energy than that of the cisisomer, with an offset of approximately $1.8 \mathrm{~kJ} / \mathrm{mol}$. Two minima exist on both the cis- and trans-conformer PESs, with dihedral angles H-C7-C5-C6 of exactly $0^{\circ}$ and $180^{\circ}$. As illustrated in Figure 2, the methyl tops of the isopropyl (C8 and C9) are out-of-plane at the minima. For both the cis and the trans curves, the lowest minima coincide with a dihedral angle of $180^{\circ}$, where the hydrogen connected to C7 is opposite to the hydroxyl group. We labeled those conformations with $\mathrm{B}$ and the second local minimum at a dihedral angle of $0^{\circ}$ with A. Hence, in total we found four conformations, two conformers each in the two conformational families of cisand trans-carvacrol.

The isomerization barrier between the A and B rotamers that corresponds to rotation of the isopropyl group with respect to the rest of the molecule was determined to be approximately $10 \mathrm{~kJ} / \mathrm{mol}$ for both cis- and trans-conformers. The barrier to cis-trans isomerization, corresponding to rotating the hydroxyl group around the $\mathrm{C} 1-\mathrm{O}$ bond, was computed to be $15 \mathrm{~kJ} / \mathrm{mol}$ for both $\mathrm{A}$ and $\mathrm{B}$ rotamers. The computational results for the barrier to internal rotation of the methyl group bonded to the $\mathrm{C} 2$ carbon are listed in Tables I and II. For the analysis of the hindered rotation, the Hamiltonian of the rigid rotor has to be extended to include the kinetic and potential energy terms of the internal rotor. In the case of a threefold symmetric top, the potential energy, $V$, can be described in terms of the internal rotation angle $\alpha$ :

$$
V(\alpha)=\frac{1}{2} V_{3}(1-\cos (3 \alpha))+\frac{1}{2} V_{6}(1-\cos (6 \alpha))+\ldots
$$

Depending on the symmetry of the molecular framework, higher order terms may also need to be included. Thus, to convert the computed barrier height into a quantity that can be compared with experimental results, the potential energy scans were fitted using Eq. (1). For a methyl group in highly symmetric local environments, e.g., as a substituent on a phenyl ring with symmetric substitution in both ortho positions, such as in thymol (discussed in Sec. III B), the $V_{6}$ contribution could be significant. For carvacrol, the single hydroxyl group substituted ortho to the methyl group breaks this symmetry so that truncation considering only the $V_{3}$ term is sufficient. As expected, the barrier heights differ significantly between the cis- and trans-isomers.

As a test case, we also calculated the internal rotation barrier of a methyl top within the isopropyl group for the trans-carvacrol-A isomer to ensure that it is too high to have

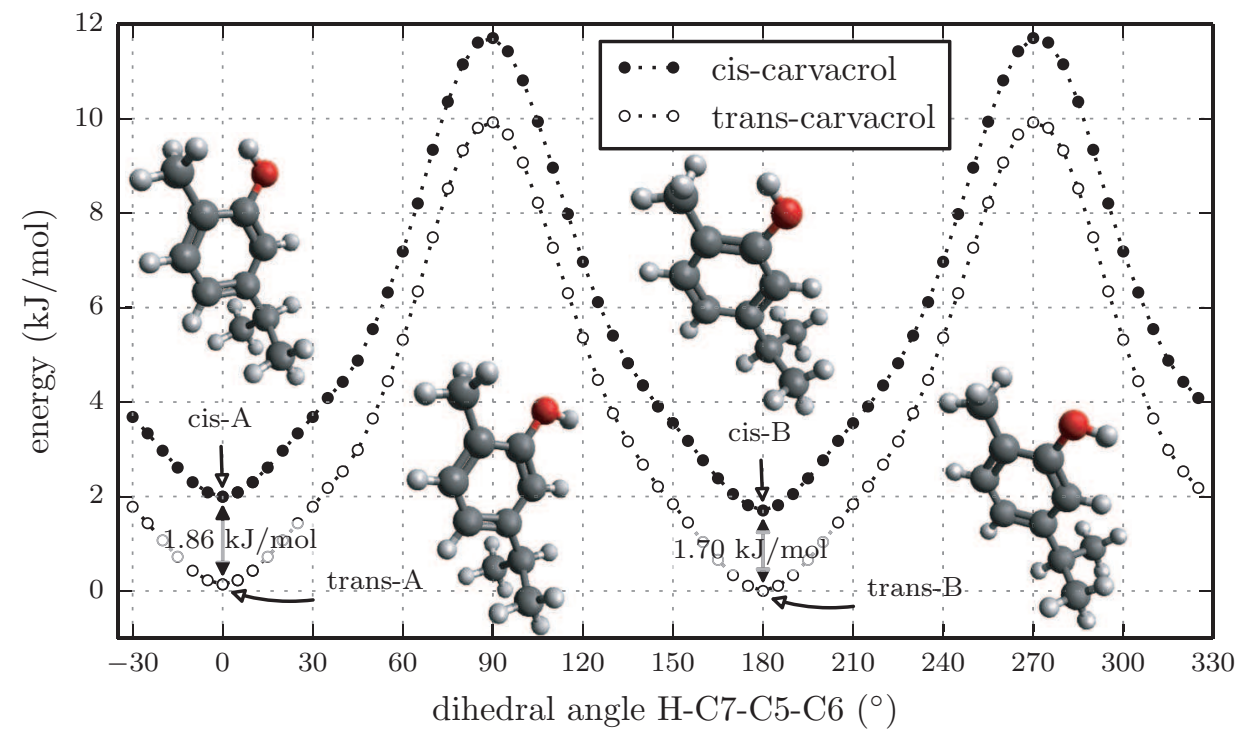

FIG. 2. Scan of the potential energy surface calculated along the H-C7-C5-C6 dihedral angle according to the labeling in Fig. 1 at the B3LYP/6-311++G(d,p) level of theory for cis- and trans-carvacrol. The position of the observed conformations (A, B) is indicated in the plot and illustrated with the corresponding structure. 
TABLE I. Rotational constants, dipole moments, and barriers to internal rotation of the $\mathrm{C} 2$ methyl group for cis-carvacrol. Note the errors given here for the measured values are standard errors. $\mathrm{N}$ is the number of lines included in the fit and $\sigma$ is the standard deviation of the fit.

\begin{tabular}{|c|c|c|c|c|c|c|}
\hline & \multicolumn{3}{|c|}{ cis-carvacrol-A } & \multicolumn{3}{|c|}{ cis-carvacrol-B } \\
\hline & Experiment & B3LYPa & $\mathrm{MP} 2^{\mathrm{b}}$ & Experiment & B3LYP & $\mathrm{MP} 2^{\mathrm{b}}$ \\
\hline $\mathrm{A}(\mathrm{MHz})$ & $2274.0616(37)$ & 2281.04 & 2265.57 & $2152.8772(44)$ & 2163.74 & 2142.48 \\
\hline $\mathrm{B}(\mathrm{MHz})$ & $643.77212(46)$ & 642.53 & 643.35 & $672.34073(50)$ & 669.87 & 672.76 \\
\hline $\mathrm{C}(\mathrm{MHz})$ & $564.76557(38)$ & 564.32 & 563.77 & $578.22275(42)$ & 577.29 & 577.62 \\
\hline$\left|\mu_{a}\right|$ (D) & $\ldots$ & 0.94 & 0.77 & $\ldots$ & 1.12 & 1.05 \\
\hline$\left|\mu_{b}\right|$ (D) & $\ldots$ & 0.94 & 1.30 & $\ldots$ & 0.80 & 1.14 \\
\hline$\left|\mu_{c}\right|$ (D) & $\ldots$ & 0.00 & 0.00 & $\ldots$ & 0.00 & 0.00 \\
\hline$V_{3}\left(\mathrm{~kJ} \mathrm{~mol}^{-1}\right)$ & $\ldots$ & $5.61^{\mathrm{c}}$ & $\ldots$ & $\ldots$ & $5.90^{\mathrm{c}}$ & $\ldots$ \\
\hline $\mathrm{N}$ & 15 & $\ldots$ & $\ldots$ & 27 & $\ldots$ & $\ldots$ \\
\hline$\sigma(\mathrm{kHz})$ & 5.5 & $\ldots$ & $\ldots$ & 8.8 & $\ldots$ & $\ldots$ \\
\hline
\end{tabular}

aug-cc-pVTZ basis set.

${ }^{\mathrm{b}} 6-311++\mathrm{G}(\mathrm{d}, \mathrm{p})$ basis set.

${ }^{\mathrm{c} B}$ B LYP/6-311++G(d,p).

an observable effect on the spectra from our experiment. The barrier for this motion and isomer should be the lowest of all of the isomers investigated here and was computed to be $13.2 \mathrm{~kJ}$ mol.

For comparison with our experimentally obtained molecular constants, the geometries of the four minima were further optimized and frequency calculations were carried out at the B3LYP/6-311++G(d,p), B3LYP/aug-cc-pVTZ, M06-2X/6-311++G(d,p), and MP2/6-311++G(d,p) levels of theory. Trans-carvacrol-B was confirmed to be the global minimum by all methods except MP2, which predicts the transcarvacrol A conformer to be the global minimum. The B3LYP calculations predict just a $0.3 \mathrm{~kJ} / \mathrm{mol}$ energy difference between the different trans-rotamers of carvacrol. The energy ordering of the cis- vs. the trans-conformers is, however, confirmed by all calculations. We present the calculated and fitted rotational constants in Tables I and II. The zero-point corrected energies are summarized in Table III.

We identified all four minimum structures discussed above in the broadband microwave spectrum of carvacrol covering 2-8.5 GHz. Figure 3 shows a fraction of the spectrum from $3600-3615 \mathrm{MHz}$, in which transitions from all four conformations can be observed. They all exhibit a-type spectra of near prolate asymmetric rotors and a few b-type transitions.

TABLE II. Rotational constants, dipole moments, and barriers to internal rotation of the $\mathrm{C} 2$ methyl group for trans-carvacrol. The errors given here for the measured values are standard errors. $\mathrm{N}$ is the number of lines included in the fit and $\sigma$ is the standard deviation of the fit. Note that the angles $(\delta, \varepsilon)$ and the rotational constant of the methyl top, $F_{0}$, are not fitted and their uncertainties are not considered in the fit. This omission mainly affects the $V_{3}$ parameter, its error, and hence the resulting error of the $V_{3}$ parameter is most likely underestimated. The direction cosines between the internal rotation axis (i) and the principal axes (a, b, c) are also given, employing the principal axis system orientation depicted in Figure 1(d).

\begin{tabular}{|c|c|c|c|c|c|c|}
\hline & \multicolumn{3}{|c|}{ trans-carvacrol-A } & \multicolumn{3}{|c|}{ trans-carvacrol-B } \\
\hline & Experiment & B3LYPa & $\mathrm{MP} 2^{\mathrm{b}}$ & Experiment & B3LYPa & $\mathrm{MP} 2^{\mathrm{b}}$ \\
\hline $\mathrm{A}(\mathrm{MHz})$ & $2283.1750(15)$ & 2288.58 & 2281.82 & $2159.0853(25)$ & 2169.50 & 2153.85 \\
\hline $\mathrm{B}(\mathrm{MHz})$ & $642.240345(30)$ & 641.17 & 641.27 & $670.84871(51)$ & 668.31 & 670.63 \\
\hline $\mathrm{C}(\mathrm{MHz})$ & $564.09631(25)$ & 563.68 & 563.13 & $577.52683(41)$ & 576.48 & 576.84 \\
\hline$\left|\mu_{a}\right|$ (D) & $\ldots$ & 0.90 & 1.28 & $\ldots$ & 1.06 & 1.42 \\
\hline$\left|\mu_{b}\right|$ (D) & $\ldots$ & 0.90 & 0.81 & $\ldots$ & 0.60 & 0.45 \\
\hline$\left|\mu_{c}\right|$ (D) & $\ldots$ & 0.00 & 0.00 & $\ldots$ & 0.00 & 0.00 \\
\hline$\rho$ & $0.0138^{\mathrm{c}}$ & 0.0138 & 0.0140 & $0.0134^{\mathrm{c}}$ & 0.0134 & 0.0135 \\
\hline$F_{0}(\mathrm{GHz})$ & $161.5^{\mathrm{d}}$ & 161.5 & 159.7 & $161.5^{\mathrm{d}}$ & 161.5 & 159.6 \\
\hline$\varepsilon(\operatorname{deg})$ & $180.0^{\mathrm{d}}$ & 180.0 & 180.0 & $180.0^{\mathrm{d}}$ & 180.0 & 178.4 \\
\hline$\delta(\operatorname{deg})$ & $13.0^{\mathrm{d}}$ & 13.0 & 12.9 & $1.02^{\mathrm{d}}$ & 1.02 & 0.35 \\
\hline$\angle(i, a)(\mathrm{deg})$ & $13.0^{\mathrm{d}}$ & 13.0 & 12.9 & $1.02^{\mathrm{d}}$ & 1.02 & 0.35 \\
\hline$\angle(i, b)(\mathrm{deg})$ & $103.0^{\mathrm{d}}$ & 103.0 & 102.9 & $91.0^{\mathrm{d}}$ & 91.0 & 90.4 \\
\hline$\angle(i, c)(\mathrm{deg})$ & $90.0^{\mathrm{d}}$ & 90.0 & 90.0 & $90.0^{\mathrm{d}}$ & 90.0 & 90.0 \\
\hline$V_{3}\left(\mathrm{~kJ} \mathrm{~mol}^{-1}\right)$ & $4.0871(25)$ & $3.7^{\mathrm{e}}$ & $\ldots$ & $4.4024(16)$ & $3.9^{\mathrm{e}}$ & $\ldots$ \\
\hline $\mathrm{N}$ & 49 & $\ldots$ & $\ldots$ & 42 & $\ldots$ & $\ldots$ \\
\hline$\sigma(\mathrm{kHz})$ & 8.7 & $\ldots$ & $\ldots$ & 10.9 & $\ldots$ & $\ldots$ \\
\hline
\end{tabular}

a aug-cc-pVTZ basis set.

${ }^{\mathrm{b}} 6-311++\mathrm{G}(\mathrm{d}, \mathrm{p})$ basis set.

${ }^{\mathrm{c}} \rho$ was not fitted but computed using the fitted rotational constants.

${ }^{\mathrm{d}}$ Values fixed to calculated values (B3LYP/aug-cc-pVTZ).

${ }^{\mathrm{e}} \mathrm{B} 3 \mathrm{LYP} / 6-311++\mathrm{G}(\mathrm{d}, \mathrm{p})$. 
TABLE III. Comparison of the zero-point corrected energies $(\mathrm{kJ} / \mathrm{mol})$ and populations of the different structural isomers of thymol and carvacrol using different methods and basis sets.

\begin{tabular}{lccccc}
\hline \hline & B3LYP $^{\mathrm{a}}$ & B3LYP $^{\mathrm{b}}$ & M06-2X $^{\mathrm{a}}$ & MP2 $^{\mathrm{a}}$ & Population $^{\mathrm{c}}$ \\
\hline Thymol & & & & & \\
trans A & 0 & 0 & 0 & 0 & 0.48 \\
trans B & 1.06 & 1.62 & 2.56 & 0.75 & 0.28 \\
cis A & 3.38 & 3.10 & 1.99 & 3.58 & 0.18 \\
cis B & 6.00 & 6.06 & 6.72 & 8.28 & 0.07 \\
Carvacrol & & & & & \\
trans A & 0.29 & 0.26 & 2.04 & 0 & 0.28 \\
trans B & 0 & 0 & 0 & 0.71 & 0.30 \\
cis A & 1.59 & 1.40 & 2.50 & 1.12 & 0.20 \\
cis B & 1.57 & 1.19 & 2.76 & 1.51 & 0.21 \\
\hline \hline
\end{tabular}

a $6-311++\mathrm{G}(\mathrm{d}, \mathrm{p})$.

${ }^{b}$ aug-cc-pVTZ.

${ }^{\mathrm{c}} \mathrm{B} 3 \mathrm{LYP} / \mathrm{aug}$-cc-pVTZ energies used for calculating the Boltzmann population, temperatures of $T=373.15 \mathrm{~K}$ and $T=413.15 \mathrm{~K}$ were used for thymol and carvacrol, respectively.

Furthermore, some transitions were split into doublets, which we ascribe to internal rotation of the methyl group connected to the phenyl ring. These splittings were small $(\approx 100 \mathrm{kHz})$ and were observed only from the two conformations with the most intense spectra. These additional spectral features give us valuable information for spectral assignment. We fit the spectra exhibiting no internal rotation splittings to a rigid rotor model using Pickett's suite of programs, SPFIT. ${ }^{31}$ The nature of the fitted transitions, at low $\mathrm{J}$, did not permit the determination of the distortion constants from the fit. The rotational constants obtained and the lower line intensities suggest the assignment of the conformers without line splittings to the cis-isomers of carvacrol. These assignments are supported by the computed internal rotation barrier heights for the $\mathrm{C} 2$ methyl group in the cis-isomers (see Table I). With barriers of $5.6 \mathrm{~kJ} / \mathrm{mol}$ and $5.9 \mathrm{~kJ} / \mathrm{mol}$, they are $50 \%$ higher than the barriers for the trans-isomers and thus should yield line splittings too narrow to be resolved in our experiments. The further assignment of the A- and B-rotamers of cis-carvacrol was straightforward because their rotational constants are sufficiently different and agree well with the calculated values. Table I shows the results of the spectral assignment of ciscarvacrol. The remaining lines in the spectrum, which were split due to internal rotation, could be assigned to the transisomers of carvacrol.

A more thorough analysis of the effect that internal rotation has on the observed spectra requires inclusion of the kinetic energy term of the internal rotor into the molecular Hamiltonian. In principle this implementation is straightforward, but different coordinate transformations have been proposed for handling the problem efficiently. ${ }^{32,33}$ Herein, we employed the internal axis method (IAM) developed by Woods, ${ }^{34,35}$ also known as the combined axis method (CAM). Besides the barrier height, $V_{3}$, the analysis of the internal rotation also provides information about the orientation of the internal rotor within the molecular framework and its moment of inertia. A threefold barrier results in splittings of the energy levels into (non degenerate) A states and doubly degenerate $\mathrm{E}$ states which vary with the rotational quantum numbers and thus leads to splittings in the spectrum.

We chose the program XIAM to perform a fit of the spectrum including transitions of both $\mathrm{A}$ and $\mathrm{E}$ symmetry. It is capable of treating up to three internal rotors with a $V_{3}$ potential and applies the internal axis method. ${ }^{36}$ The orientation of the methyl rotor within the principal axis system is specified using the angles $\delta$ and $\varepsilon$. In the case of a prolate asymmetric top using the $I^{r}$-representation, $\delta$ describes the angle between the $a$-rotational axis of the molecule and the axis of internal rotation. The angle $\varepsilon$ describes the angle between the $b$-rotational axis and the projection of the internal rotor axis onto the xy-plane of the principal axis system of the molecule.

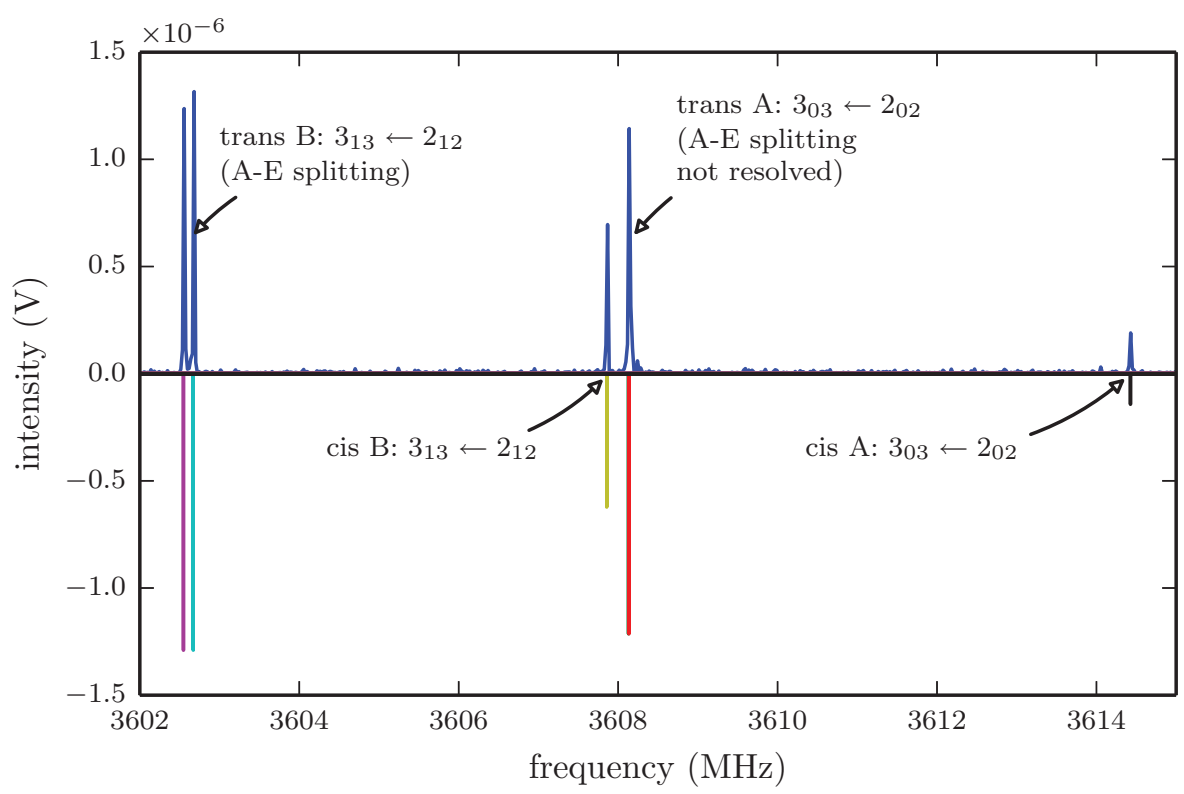

FIG. 3. Part of the microwave spectrum of carvacrol in the region of $3600-3615 \mathrm{MHz}$. The upper trace shows the measured spectrum, the lower trace is a simulation based on fitted molecular parameters. The small line splitting due to internal rotation is apparent for the $3_{13} \leftarrow 2_{12}$ transition of trans-carvacrol-B. In comparison, the smaller line splitting of the transition $3_{03} \leftarrow 2_{02}$ of trans-carvacrol-A was not resolved. No line splitting was observed for the cis-conformers. 
The structure calculations for trans-carvacrol indicate that the internal rotation axis almost coincides with the $a$ rotational axis. This result implies that $\delta$ is close to $0^{\circ}$ while $\varepsilon$ is only poorly defined. Hence, fits of the data including these angles gave unphysical or diverging results. Therefore, the angles and the rotational constant of the methyl top $\left(F_{0}\right)$ were held fixed at the calculated values and only the rotational constants of the molecule and the barrier to internal rotation were allowed to float. The results of these fits are summarized in Table II. As for the cis-rotamers, the assignment of the transrotamers to trans-carvacrol-A or B was also unambiguous by comparison of the measured and the calculated rotational constants. In total we assigned 49 lines to trans-carvacrol-A and 42 lines to trans-carvacrol-B.

\section{B. Thymol}

Due to their structural similarities, we followed the same analysis protocol for thymol as for carvacrol. Therefore, we mainly focus on the differences between the molecules rather than the analysis details. The relaxed potential energy scan of the internal rotation of the isopropyl group already reveals significant differences compared to carvacrol. As shown in Figure 4, the cuts through the potential energy surfaces exhibit three minima of trans- and cis-thymol instead of two as observed for carvacrol. For the A rotamers, the hydrogen at C7 is slightly out of plane, resulting in two equivalent minima. Compared to carvacrol, the energy difference between the cis- and the trans-conformational families of thymol is much larger (approximately a factor of two and four larger for the A and B rotamers, respectively) because of the additional structural stabilization from the hydrogen bonds formed in the trans-thymol rotamers. The energy ordering of rotamers $\mathrm{A}$ and $\mathrm{B}$ is also reversed for thymol as compared to carvacrol at the B3LYP/6-311++G(d,p) level of calculation. Finally, the potential barrier between rotamers $\mathrm{A}$ and $\mathrm{B}$ was determined to be around $23 \mathrm{~kJ} / \mathrm{mol}, 2.3$-times higher than in carvacrol.
These results are expected because the hydroxyl group in thymol is able to more strongly interact with the isopropyl group than in carvacrol. The barrier of the cis-trans interconversion by rotating the hydroxyl group around the $\mathrm{C} 1-\mathrm{O}$ bond was calculated to be $15.4 \mathrm{~kJ} / \mathrm{mol}$ and $16.1 \mathrm{~kJ} / \mathrm{mol}$ for the rotamers $\mathrm{A}$ and $\mathrm{B}$, respectively.

The structure of thymol also suggests a lower barrier to internal rotation for the methyl group at the carbon 5. This hypothesis was confirmed by calculations on all four conformations, which yielded barrier heights between $0.04 \mathrm{~kJ} / \mathrm{mol}$ (cis-thymol) and $0.3 \mathrm{~kJ} / \mathrm{mol}$ (trans-thymol), values at least ten times smaller than for carvacrol. Therefore, we expect an obvious effect of methyl group internal rotation on the microwave spectra of all conformations of thymol. The calculated barriers were fit using Eq. (1) and are summarized in Table IV. Even though the $V_{6}$ part of the potential is negligible for trans-thymol, it becomes significant for $c i s$-thymol, and so we included both the $V_{3}$ and $V_{6}$ terms in Table $\mathrm{V}$. This result is an indication that the interaction of the hydroxyl group with the methyl group becomes weaker so that the local environment of the methyl rotor at carbon 5 approaches $C_{2 V}$ symmetry, as in toluene. To exclude possible effects on the spectra by the rotation of one of the isopropyl methyl groups, we evaluated a methyl group rotation for trans-thymol-A. It is very unlikely that the calculated barrier height of $13.7 \mathrm{~kJ} / \mathrm{mol}$ will impact the spectrum in a measurable way.

The analysis of the spectrum revealed three conformers, with one having much higher intensity than the other two. The spectra are dominated by a-type transitions, which are not so apparent in the overview spectrum in Figure 5 due to widely split lines from internal rotation of the methyl group. We first concentrate on the analysis of the highest intensity conformer. For this species, transition splittings due to methyl group rotation could be identified and were successfully assigned. The smaller internal rotation barrier compared to carvacrol increased the line splitting up to $88 \mathrm{MHz}$. We were able to assign 39 and 15 a-type lines of A and E symmetry,

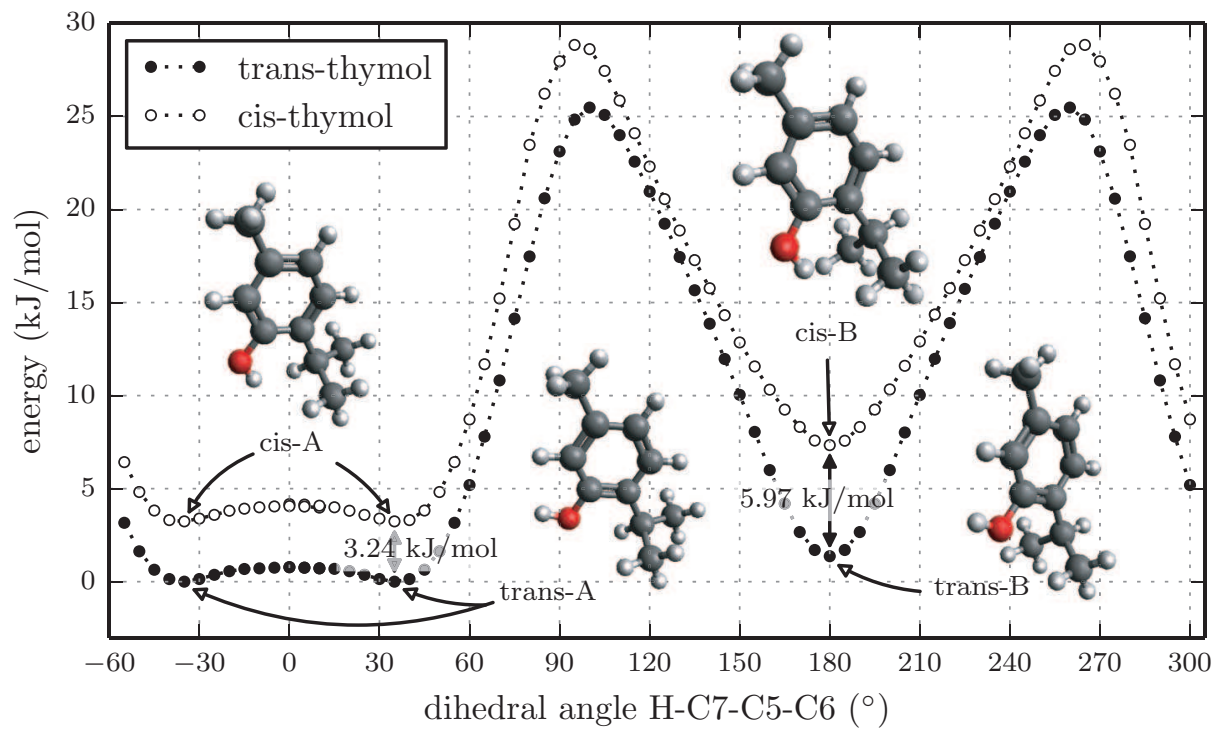

FIG. 4. Scan of the potential energy surface calculated along the H-C7-C2-C1 dihedral angle at the B3LYP/6-311++G(d,p) level of theory for cis- and transthymol. The two minima of cis-thymol-A are equivalent, as are the two for trans-thymol-A. We assume that the structure of trans-thymol-A is stabilized by a hydrogen bond. 
TABLE IV. Rotational constants, dipole moments, and barriers to internal rotation of the C5 methyl group for trans-thymol. The errors given here for the measured values are standard errors. $\mathrm{N}$ is the number of lines included in the fit and $\sigma$ is the standard deviation of the fit. The direction cosines between the internal rotation axis (i) and the principal axes (a, b, c) are also given, employing the principal axis system orientation depicted in Figure 1(d).

\begin{tabular}{|c|c|c|c|c|c|c|}
\hline & \multicolumn{3}{|c|}{ trans-thymol-A } & \multicolumn{3}{|c|}{ trans-thymol-B } \\
\hline & Experiment & B3LYPa & $\mathrm{MP}^{\mathrm{b}}$ & Experiment & B3LYPa & $\mathrm{MP} 2^{\mathrm{b}}$ \\
\hline $\mathrm{A}(\mathrm{MHz})$ & $2034.068(71)$ & 2039.45 & 2024.06 & $2261.070(62)$ & 2237.63 & 2229.72 \\
\hline $\mathrm{B}(\mathrm{MHz})$ & $739.18202(35)$ & 735.91 & 738.22 & $708.92030(45)$ & 707.59 & 708.94 \\
\hline $\mathrm{C}(\mathrm{MHz})$ & $591.51673(30)$ & 591.73 & 593.03 & $611.86246(50)$ & 611.13 & 611.43 \\
\hline$\left|\mu_{a}\right|$ (D) & $\ldots$ & 1.12 & 1.43 & $\ldots$ & 1.15 & 1.50 \\
\hline$\left|\mu_{b}\right|(\mathrm{D})$ & $\ldots$ & 0.72 & 0.53 & $\ldots$ & 0.82 & 0.72 \\
\hline$\left|\mu_{c}\right|$ (D) & $\ldots$ & 0.10 & 0.07 & $\ldots$ & 0.00 & 0.04 \\
\hline$D_{p i 2 J}(\mathrm{kHz})$ & $2.44(23)$ & $\ldots$ & $\ldots$ & $\ldots$ & $\ldots$ & $\ldots$ \\
\hline$\rho$ & $0.012990(42)$ & 0.0126 & 0.0126 & $\ldots$ & 0.0137 & 0.0138 \\
\hline$F_{0}(\mathrm{GHz})$ & $156.26(51)$ & 161.7 & 160.1 & $\ldots$ & 161.7 & 160.1 \\
\hline$\varepsilon(\mathrm{deg})$ & $111.73(16)$ & 112.4 & 105.2 & $\ldots$ & 0.0 & 7.1 \\
\hline$\delta(\mathrm{deg})$ & $3.8471(46)$ & 3.5 & 4.6 & $\ldots$ & 8.3 & 8.8 \\
\hline$\angle(i, a)(\mathrm{deg})$ & $3.8471(46)$ & 3.5 & 4.6 & $\ldots$ & 8.3 & 8.8 \\
\hline$\angle(i, b)(\mathrm{deg})$ & $91.423(12)$ & 91.3 & 91.2 & $\ldots$ & 81.7 & 81.2 \\
\hline$\angle(i, c)(\mathrm{deg})$ & $86.4267(84)$ & 86.8 & 85.5 & $\ldots$ & 90.0 & 88.9 \\
\hline$V_{3}\left(\mathrm{~kJ} \mathrm{~mol}^{-1}\right)$ & $0.3699(11)$ & $0.318^{c}$ & $\ldots$ & $\ldots$ & $0.261^{\mathrm{c}}$ & $\ldots$ \\
\hline $\mathrm{N}$ & 54 & $\ldots$ & $\ldots$ & 12 & $\ldots$ & $\ldots$ \\
\hline$\sigma(\mathrm{kHz})$ & 11.6 & $\ldots$ & $\ldots$ & 4.0 & $\ldots$ & $\ldots$ \\
\hline
\end{tabular}

${ }^{\mathrm{a}}$ aug-cc-pVTZ basis set.

${ }^{\mathrm{b}} 6-311++\mathrm{G}(\mathrm{d}, \mathrm{p})$ basis set

${ }^{c} B 3 L Y P / 6-311++G(d, p)$.

respectively. For the b-type lines, we only observed those of A symmetry. We performed a global fit including all lines using $\mathrm{XIAM}$, in which the angles $\delta$ and $\varepsilon$ and the rotational constant of the methyl rotor, $F_{0}$, could be fit in addition to the rotational constants and barrier height. Only the $V_{3}$ part of the potential was retained in the final fit, because, as stated before, the calculations suggest that the $V_{6}$ term is negligible (less than $3 \%$ compared to the $V_{3}$ term). Furthermore, including $V_{6}$ in the fit did not improve it and led to unphysical results for $V_{3}$ and $V_{6}$. Moreover, we also included the internal rotation-overall rotation distortion operator, $D_{p i 2 J}$, which led to better fits, especially for lines with $K_{a}>1$. This more complete assessment for thymol compared to carvacrol was possible due to the larger line splitting, so that more lines of E symmetry could be assigned. Even though the fit did not diverge, the resulting values of the internal rotor angles $\delta$ and $\varepsilon$ were dependent on the starting guess values of the fit. Therefore, we used the calculated angles as starting values, which are assumed to be already close to the real values. The convergence of all other constants included in the fit was much more robust. Because of the structural similarities of the cis- and trans-isomers, an unambiguous assignment to the calculated structures based on only the rotational constants is not possible. However, the energy ordering of the different conformations (Table III) and the barrier height to internal rotation provide sufficient information for a solid assignment. Taking

TABLE V. Rotational constants and dipole moments for cis-thymol. Only cis-thymol-A lines were identified in the spectra, and thus only experimental values for this cis conformation of thymol could be determined. The errors given here for the measured values are standard errors. $\mathrm{N}$ is the number of lines included in the fit and $\sigma$ is the standard deviation of the fit.

\begin{tabular}{|c|c|c|c|c|c|}
\hline & \multicolumn{3}{|c|}{ cis-thymol-A } & \multicolumn{2}{|c|}{ cis-thymol-B } \\
\hline & Experiment & $\mathrm{B} 3 \mathrm{LYP}^{\mathrm{a}}$ & $\mathrm{MP} 2^{\mathrm{b}}$ & $\mathrm{B} 3 \mathrm{LYP}^{\mathrm{a}}$ & $\mathrm{MP}^{\mathrm{b}}$ \\
\hline $\mathrm{A}(\mathrm{MHz})$ & $2048.642(32)$ & 2027.07 & 2009.27 & 2218.7704 & 2205.61 \\
\hline B (MHz) & $739.25463(50)$ & 735.71 & 739.12 & 704.57 & 705.10 \\
\hline $\mathrm{C}(\mathrm{MHz})$ & $590.31448(44)$ & 591.02 & 591.56 & 608.31 & 607.78 \\
\hline$\left|\mu_{a}\right|$ (D) & $\ldots$ & 1.25 & 1.15 & 1.11 & 0.96 \\
\hline$\left|\mu_{b}\right|$ (D) & $\cdots$ & 0.72 & 1.11 & 1.01 & 1.35 \\
\hline$\left|\mu_{c}\right|$ (D) & $\ldots$ & 0.04 & 0.14 & 0.03 & 0.36 \\
\hline$V_{3}\left(V_{3}, V_{6}\right)\left(\mathrm{kJ} \mathrm{mol}^{-1}\right)$ & $\cdots$ & $0.106(0.069,0.057)^{\mathrm{c}}$ & $\ldots$ & $0.036(0.025,0016)^{\mathrm{c}}$ & $\ldots$ \\
\hline $\mathrm{N}$ & 10 & $\ldots$ & $\ldots$ & $\ldots$ & $\ldots$ \\
\hline$\sigma(\mathrm{kHz})$ & 4.4 & $\ldots$ & $\ldots$ & $\ldots$ & $\ldots$ \\
\hline
\end{tabular}

a aug-cc-pVTZ basis set.

${ }^{\mathrm{b}} 6-311++\mathrm{G}(\mathrm{d}, \mathrm{p})$ basis set.

${ }^{c}$ B3LYP/6-311++G(d,p). 


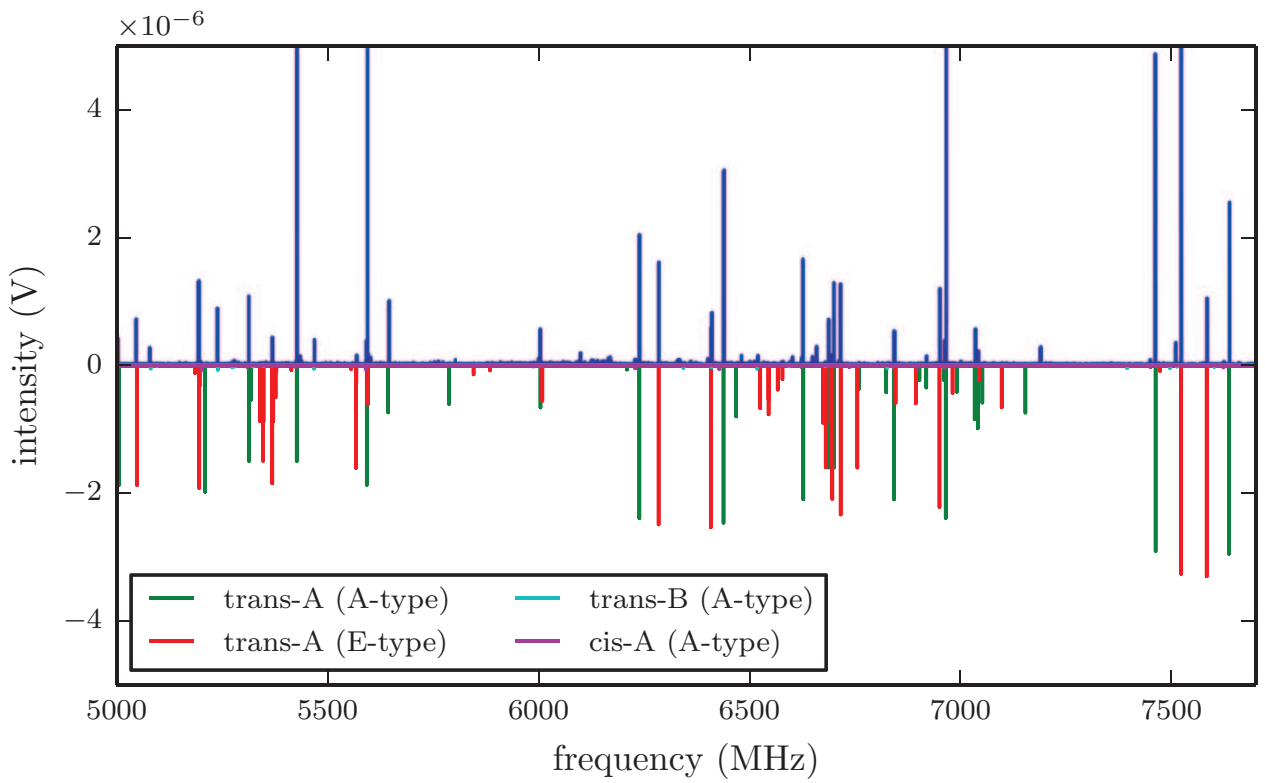

FIG. 5. A part of the broadband microwave spectrum of thymol in the frequency region of 5.0-7.7 GHz. It is dominated by the A and E species components of trans-thymol A. The transitions of trans-thymol B and cis-thymol A are much weaker than those of trans-thymol A.

this information into account, the highest intensity conformer must be the trans-thymol-A conformer.

As already mentioned, the line intensities of the other two conformers were much weaker and sometimes even barely distinguishable from the noise $(\mathrm{S} / \mathrm{N}$ ratio is around $2: 1$ for the lower intensity lines). Nevertheless, 10 lines were identified for one of the conformers and 12 lines for the other one. Only a-type transitions of A symmetry could be assigned for both of them. Even though there was evidence for line splitting as the calculated barrier to internal rotation predicts, it was not possible to clearly identify any E symmetry lines. The comparison between the fitted and calculated rotational constants suggests that one of these conformations is cis-thymolA, while the calculated energy ordering (Table III) suggests that the other one is trans-thymol-B.

\section{DISCUSSION}

The measurements and calculations revealed that the energy differences among the conformers of thymol are larger than for carvacrol. This spread in energies explains the presence of four conformations in the supersonic expansion for carvacrol, compared to only three for thymol. The comparison of the zero-point corrected energies as well as the calculated populations of the different structural isomers given in Table III further elucidates this result. The calculated energy of the cis-thymol-B conformer, which was not observed in the spectrum, is at least two times higher than the second highest energy conformer, cis-thymol-A, for all methods and basis sets.

The influence of the substituents on the barrier to methyl rotation was investigated previously in various substituted toluenes. $^{23-25,37-39}$ Some of the results are listed in Table VI together with our thymol and carvacrol results. This list shows some clear trends for internal rotation barriers. The barrier heights of trans-carvacrol and anti-o-cresol are very similar because the molecules only differ in that cresol does not contain an isopropyl substituent. We were not able to resolve the effects of the internal rotation for cis-carvacrol, but the barrier height can be expected to be similar to that of $s y n$-o-cresol. A direct comparison of the barrier heights of trans-thymol and syn- $m$-cresol was not possible, because Hellweg et al. also included the $V_{6}$ term of the potential, ${ }^{25}$ which we did not do because it resulted in unphysical results. However, the $V_{3}$ contribution to the potential is of the same order of magnitude. In summary, the barrier height of the methyl rotor seems to depend mainly on steric effects, as also suggested by Gerhard et al. ${ }^{37}$ Hence, more extended substituents close to the rotating methyl group, such as another methyl group (e.g., as in xylene), result in higher barriers than more compact groups, such as a cyano group (e.g., as in $o$-tolunitrile). For a hydroxyl group, the barrier height very much depends on the orientation of the hydrogen, as expected.

TABLE VI. Comparison of the barriers to internal rotation of thymol, carvacrol, and similar molecules. The barrier heights are listed in increasing order.

\begin{tabular}{lc}
\hline \hline & $V_{3}(\mathrm{~kJ} / \mathrm{mol})$ \\
\hline Toluene & $V_{6}=0.05787337(11)^{23}$ \\
anti-m-cresol & $0.038(2)\left(V_{6}=-0.16(2)\right)^{25}$ \\
syn- $m$-cresol & $0.2684(8)\left(V_{6}=-0.13(1)\right)^{25}$ \\
trans-thymol-A & $0.3699(11)($ this work $)$ \\
$o$-tolunitrile & $2.24538(4)^{38}$ \\
trans-carvacrol-A & $4.0875(25)($ this work $)$ \\
trans-carvacrol-B & $4.4025(16)($ this work $)$ \\
anti-o-cresol & $4.4256(14)^{24}$ \\
$o$-chlorotoluene & $5.527(10)^{37}$ \\
memphenesin (conf. A) & $5.736(18)^{40}$ \\
$o$-xylene & $6.23(21)^{39}$ \\
syn-o-cresol & $7.912(46)^{24}$ \\
\hline \hline
\end{tabular}




\section{CONCLUSION AND OUTLOOK}

We presented the microwave spectra of two monoterpenoids, thymol and carvacrol. Four conformations of carvacrol were identified in the cold conditions of the molecular beam. In contrast, only three conformations of thymol were present. This difference can be explained by the higher energies of the group of cis-conformers of thymol relative to the global minimum trans-conformer compared with carvacrol, where the energy differences between the cis- and trans-conformer groups are smaller.

For thymol and carvacrol, we observed internal rotation of the terminal methyl top and obtained a fitted barrier height that is consistent with measurements of similar molecules. The one order of magnitude difference in the barrier heights observed for these two molecules can be directly ascribed to the different positions of the hydroxyl substituent on the aromatic ring. In carvacrol, the hydroxyl group is substituted ortho to the methyl top and thus strong interactions arise, resulting in a high barrier to internal rotation. In thymol, the hydroxyl group is substituted meta to the methyl top and thus the larger distance between these groups results in weaker hindrance to methyl rotation and thus a lower internal rotation barrier.

The results of the quantum chemical calculations agreed very well with the experiment. The rotational constants from both the MP2- and B3LYP-based methods are near to the values obtained experimentally, indicating that they are accurate enough to model the structures of the kinds of molecular systems investigated here.

In future work, extensions to studies of secondary interactions, i.e., hydrogen bonding by substitution of a second hydroxyl group onto the aromatic ring, will be interesting. We are also interested in investigating chiral molecules such as the related monoterpenoid, menthol. It exhibits the same substituent patterns as thymol, but has a central cyclohexane ring instead of a phenyl ring. The additional structural flexibility of menthol influences the number of potential low-energy conformers and it will be intriguing to draw direct comparisons to planar thymol (see supplementary material ${ }^{41}$ ).

\section{ACKNOWLEDGMENTS}

The authors wish to thank Alberto Lesarri for fruitful discussions and his meaningful input. D.S. acknowledges use of the octowolf computing cluster of the research group of Wolfgang Jäger at the University of Alberta, Canada. Funding of the Deutsche Forschungsgemeinschaft is acknowledged. M.S. acknowledges financial support from the Fonds der Chemischen Industrie via a Dozentenstipendium. B.M.G. acknowledges support from the CONSOLIDER Grant No. CSD 2009-00038 and the excellence cluster, "The Hamburg Centre for Ultrafast Imaging - Structure, Dynamics, and Control of Matter at the Atomic Scale," of the Deutsche Forschungsgemeinschaft for travel support.

\footnotetext{
${ }^{1}$ A. P. Bateman, S. A. Nizkorodov, J. Laskin, and A. Laskin, Phys. Chem. Chem. Phys. 13, 12199 (2011).

${ }^{2}$ M. Kanakidou, J. H. Seinfeld, S. N. Pandis, I. Barnes, F. J. Dentener, M. C. Facchini, R. Van Dingenen, B. Ervens, A. Nenes, C. J. Nielsen, E. Swi-
}

etlicki, J. P. Putaud, Y. Balkanski, S. Fuzzi, J. Horth, G. K. Moortgat, R. Winterhalter, C. E. L. Myhre, K. Tsigaridis, E. Vignati, E. G. Stephanou, and J. Wilson, Atmos. Chem. Phys. 5, 1053 (2005).

${ }^{3}$ H. V. L. Nguyen, H. Mouhib, S. Klahm, W. Stahl, and I. Kleiner, Phys. Chem. Chem. Phys. 15, 10012 (2013).

${ }^{4}$ J. R. Avilés Morenô, F. Partal Ureña, J. J. López González, and T. R. Huet, Chem. Phys. Lett. 473, 17 (2009).

${ }^{5}$ J. R. Avilés Moreno, T. R. Huet, and J. J. López González, Struct. Chem. 24, 1163 (2012).

${ }^{6}$ E. J. Cocinero, A. Lesarri, P. Écija, F. J. Basterretxea, J.-U. Grabow, J. A. Fernández, and F. Castaño, Angew. Chem., Int. Ed. 51, 3119 (2012).

${ }^{7}$ A. Lesarri, S. Mata, E. J. Cocinero, S. Blanco, J. C. López, and J. L. Alonso, Angew. Chem., Int. Ed. 41, 4673 (2002).

${ }^{8}$ A. Lesarri, E. J. Cocinero, J. C. López, and J. L. Alonso, Angew. Chem., Int. Ed. 43, 605 (2004).

${ }^{9}$ S. Blanco, A. Lesarri, J. C. López, and J. L. Alonso, J. Am. Chem. Soc. 126, 11675 (2004)

${ }^{10}$ J. L. Alonso, I. Peña, J. C. López, and V. Vaquero, Angew. Chem., Int. Ed. 48, 6141 (2009).

${ }^{11}$ J. L. Alonso, V. Vaquero, I. Peña, J. C. López, S. Mata, and W. Caminati, Angew. Chem., Int. Ed. 52, 2331 (2013).

${ }^{12}$ C. Cabezas, M. Varela, I. Peña, J. C. López, and J. L. Alonso, Phys. Chem. Chem. Phys. 14, 13618 (2012).

${ }^{13}$ I. Peña, A. M. Daly, C. Cabezas, S. Mata, C. Bermúdez, A. Niño, J. C. Lopéz, J.-U. Grabow, and J. L. Alonso, J. Phys. Chem. Lett. 4, 65 (2013).

${ }^{14}$ M. Varela, C. Cabezas, J. C. López, and J. L. Alonso, J. Phys. Chem. A 117, 13275 (2013).

${ }^{15}$ N. A. Seifert, D. P. Zaleski, C. Pérez, J. L. Neill, B. H. Pate, M. VallejoLópez, A. Lesarri, E. J. Cocinero, F. Castaño, and I. Kleiner, Angew. Chem., Int. Ed. 53, 3210 (2014).

${ }^{16}$ W.-X. Du, C. W. Olsen, R. J. Avena-Bustillos, T. H. McHugh, C. E. Levin, and M. Friedman, J. Agric. Food Chem. 56, 3082 (2008).

${ }^{17}$ J. Xu, F. Zhou, B.-P. Ji, R.-S. Pei, and N. Xu, Lett. Appl. Microbiol. 47, 174 (2008).

${ }^{18}$ A. Ben Arfa, S. Combes, L. Preziosi-Belloy, N. Gontard, and P. Chalier, Lett. Appl. Microbiol. 43, 149 (2006).

${ }^{19}$ P. C. Braga, M. Dal Sasso, M. Culici, T. Bianchi, L. Bordoni, and L. Marabini, Pharmacology 77, 130 (2006).

${ }^{20}$ M. A. Numpaque, L. A. Oviedo, J. H. Gil, C. M. García, and D. L. Durango, Trop. Plant Pathol. 36, 3 (2011).

${ }^{21}$ M. Schnell, U. Erlekam, P. R. Bunker, G. von Helden, J.-U. Grabow, G. Meijer, and A. van der Avoird, Angew. Chem., Int. Ed. 52, 5180 (2013).

${ }^{22}$ V. A. Shubert, D. Schmitz, and M. Schnell, Mol. Phys. 111, 2189 (2013).

${ }^{23}$ V. V. Ilyushin, Z. Kisiel, L. Pszczólkowski, H. Mäder, and J. T. Hougen, J. Mol. Spectrosc. 259, 26 (2010).

${ }^{24}$ A. Welzel, A. Hellweg, I. Merke, and W. Stahl, J. Mol. Spectrosc. 215, 58 (2002).

${ }^{25}$ A. Hellweg, C. Hättig, I. Merke, and W. Stahl, J. Chem. Phys. 124, 204305 (2006).

${ }^{26}$ D. Schmitz, V. A. Shubert, T. Betz, and M. Schnell, J. Mol. Spectrosc. 280, 77 (2012).

${ }^{27}$ S. T. Shipman, J. L. Neill, R. D. Suenram, M. T. Muckle, and B. H. Pate, J. Phys. Chem. Lett. 2, 443 (2011).

${ }^{28}$ W. Caminati, in Handbook of High-Resolution Spectroscopy (John Wiley \& Sons, Ltd., 2011)

${ }^{29}$ M. J. Frisch, G. W. Trucks, H. B. Schlegel et al., Gaussian 03, Revision E.01, Gaussian, Inc., Wallingford, CT, 2004.

${ }^{30}$ M. J. Frisch, G. W. Trucks, H. B. Schlegel et al., Gaussian 09, Revision B.01, Gaussian, Inc., Wallingford, CT, 2009.

${ }^{31}$ H. M. Pickett, J. Mol. Spectrosc. 148, 371 (1991).

${ }^{32}$ C. C. Lin and J. D. Swalen, Rev. Mod. Phys. 31, 841 (1959).

${ }^{33}$ I. Kleiner, J. Mol. Spectrosc. 260, 1 (2010).

${ }^{34}$ R. C. Woods, J. Mol. Spectrosc. 22, 49 (1967)

${ }^{35}$ R. C. Woods, J. Mol. Spectrosc. 21, 4 (1966).

${ }^{36}$ H. Hartwig and H. Dreizler, Z. Naturforsch. 51a, 923 (1996).

${ }^{37}$ D. Gerhard, A. Hellweg, I. Merke, W. Stahl, M. Baudelet, D. Petitprez, and G. Wlodarczak, J. Mol. Spectrosc. 220, 234 (2003).

${ }^{38}$ N. Hansen, H. Mäder, and T. Bruhn, Mol. Phys. 97, 587 (1999).

${ }^{39}$ H. Rudolph, K. Walzer, and I. Krutzig, J. Mol. Spectrosc. 47, 314 (1973).

${ }^{40}$ P. Écija, L. Evangelisti, M. Vallejo, F. J. Basterretxea, A. Lesarri, F. Castaño, W. Caminati, and E. J. Cocinero, J. Phys. Chem. B 118, 5357 (2014).

${ }^{41}$ See supplementary material at http://dx.doi.org/10.1063/1.4887337 for each molecule and conformation investigated, the line lists of the transition frequencies obtained from the spectra. 